\title{
Urbanismo a la carta: teorías, políticas, programas y otras recetas urbanas para ciudades latinoamericanas
}

\author{
A la carte urbanism: theories, policies, programs \\ and other urban recipes for Latin American cities
}

Victor Delgadillo

\begin{abstract}
Resumen
En las últimas décadas diversas ciudades latinoamericanas han impulsado un conjunto de similares políticas públicas, programas urbanos y otras "recetas" urbanísticas para: confrontar distintas problemáticas urbanas, generar competitividad económica o construir una buena imagen de la ciudad. Este conjunto de acciones parece constituir un "menú" de "recetas probadas" en distintos contextos urbanos para confrontar "con éxito" algunos problemas y desafíos urbanos. Este artículo revisa sucintamente la circulación de paradigmas y políticas urbanas; compara algunas políticas y programas urbanos realizados recientemente en Buenos Aires, Ciudad de México y Quito e intenta responder algunas preguntas: Porqué alcaldes con orientaciones políticas tan diferentes ejecutan el mismo tipo de políticas urbanas: hay una visión pragmática compartida, coincidencia política, coacción económica o una ideología dominante?
\end{abstract}

Palabras clave: Urbanismo a la carta, teoría urbana latinoamericana, políticas urbanas, circulación de paradigmas urbanos, espacio público

\begin{abstract}
In recent decades, many Latin American cities have launched a similar set of public policies, urban programs and other urban development "recipes" in order to: confront different urban problems, generate economic competitiveness or build a good city image. These actions appear to build a "menu of tested recipes" in different urban contexts to "successfully" confront different urban problems and challenges. This article briefly reviews the circulation of urban paradigms and policies, compares some urban policies and programs which have been recently conducted in Buenos Aires, Mexico City and Quito, and attempts to answer some questions: Why do mayors with different political orientations implement the same type of urban policies? Is there a shared pragmatic vision, a political coincidence, economic coercion or a dominant ideology?
\end{abstract}

Keywords: a la carte planning; Latin American urban theory; urban policies; circulation of urban paradigms; public space. 


\section{Introducción}

En las últimas décadas diversas ciudades latinoamericanas, gobernadas por partidos con la más distinta orientación política, han impulsado un conjunto de similares políticas públicas, programas urbanos y otras "recetas" urbanísticas para: 1) confrontar distintas problemáticas urbanas, 2) generar competitividad económica o 3) construir una buena imagen de la ciudad. Este conjunto de políticas, programas y recetas urbanas abarca la recuperación de los centros históricos, la introducción de carriles confinados para el uso de bicicletas y sistemas de transporte colectivo rápido, el mejoramiento de barrios y de vivienda, becas para población vulnerable, presupuestos participativos, "playas urbanas", etcétera. Se trata de un conjunto de políticas y acciones urbanas que parecen constituir un "menú" de "recetas probadas" en distintos contextos urbanos para confrontar "con éxito" algunos problemas y desafíos urbanos.

Este artículo reconoce positivamente que en América Latina hay un sistema de vasos comunicantes que históricamente han permitido el intercambio y la difusión de políticas públicas y experiencias urbanas, que han facilitado a las ciudades, sus ciudadanos y gobiernos confrontar con dignidad y eficacia diversos problemas y desafíos urbanos. La difusión de experiencias urbanas desarrolladas en otros contextos geográficos y en otros momentos históricos son condiciones básicas para la innovación urbana y el aprendizaje mutuo. Por ello, en nuestro quehacer profesional promovemos la difusión crítica de las Ilamadas "buenas" políticas que adaptadas a las condiciones locales pueden coadyuvar a la solución de problemas específicos. Sin embargo, en la difusión y reproducción de las políticas, programas y acciones urbanas que conforman este "Urbanismo a la carta" actual, destacan dos cosas: 1) el papel (más o menos impositivo) desempeñado por los organismos internacionales de financiamiento como el Banco Mundial y en nuestra región el Banco Interamericano de Desarrollo; y 2) una visión pro empresarial que a nombre de la creación de riqueza y empleo, privilegia la actividad económica directa e indirecta. ${ }^{1}$

Este artículo 1) se propone abrir nuevas perspectivas en el estudio comparado de algunas políticas públicas que simultáneamente se aplican en diversas ciudades latinoamericanas y 2) intenta responder una serie de preguntas: Qué es lo que hace que alcaldes y gobiernos locales con orientaciones políticas tan diferentes ejecuten el mismo tipo de políticas urbanas (más y/o menos neoliberales), como la introducción de "playas urbanas", circuitos confinados para bicicletas y metrobuses, o la programación de múltiples eventos culturales en espacios públicos que al parecer tienen la intención de erosionar la dimensión política y ciudadana de esos lugares para convertirlos en sitios de entretenimiento para consumidores. ¿La promoción de la misma política y el mismo tipo de acciones urbanas en distintos contextos responde a una misma visión pragmática, una coincidencia política, una coacción económica, o hay detrás de ello una teoría y una ideología dominante que soporte esa toma de decisiones? Los programas y recetas urbanas que más se difunden ¿Promueven soluciones de fondo o son simples paliativos para algunos 
problemas urbanos? ¿En ausencia de pan se trata de llevar circo a esas ciudades? ¿Se atienden necesidades de la población o se favorece la realización de negocios privados?

Este artículo analiza algunas políticas públicas en tres ciudades latinoamericanas (Buenos Aires, Quito y Ciudad de México) que han sido elegidas en función de trabajos previos (Delgadillo, 2011). A la manera de una carta de restaurante este artículo propone analizar una variedad de acciones, programas, políticas, proyectos y megaproyectos urbanos, así como estrategias de marketing urbano para distintos gustos y precios, lo que abarca la "cocina" típica, regional e internacional. El "Urbanismo a la Carta" abarca acciones puntuales, planes estratégicos; la realización de megaproyectos; la recuperación de centros históricos; así como acciones de marketing urbano (con la adopción de slogans públicos similares: Ciudad Verde, Ciudad global, Ciudad compacta, Ciudad sustentable, equitativa y competitiva; etcétera). Se trata de un "menú" con diversificado origen de esas "recetas" urbanas, cuyo análisis debe abarcar el papel desempeñado por los consultores foráneos (algunos de ellos promotores del "modelo Barcelona" for export), las agencias de cooperación internacional y los profesionistas locales (en su papel de académicos, consultores o funcionarios públicos) en la difusión de este "Urbanismo a la carta". Por cuestión de extensión en este artículo sólo analizamos las políticas del espacio público y de transporte público en bicicleta y en menor medida las políticas de mejoramiento barrial.

Tenemos conciencia que algunos aspectos de este artículo son bastante obvios y conocidos, pero creemos que analizados en el conjunto de tres ciudades Ilamaremos la atención sobre la necesidad de teorizar sobre la reproducción de estas prácticas. En síntesis, este texto pretende evidenciar las cosas en común que tienen tres políticas urbanas recientes en tres ciudades latinoamericanas, y cómo éstas son modeladas por la doctrina neoliberal, lo que alcanza a gobiernos de las más diversas orientaciones políticas (en el discurso). El artículo presenta, en la primera parte, una revisión sucinta sobre la circulación de paradigmas y políticas urbanas. El apartado dos presenta una comparación de tres políticas urbanas realizadas en las dos últimas décadas en las tres ciudades objeto de estudio. El último apartado presenta las conclusiones y una agenda de temas para seguir investigando sobre la teoría urbana que subyace a una serie de políticas públicas urbanas que se realizan en más de una ciudad latinoamericana.

\section{Sobre la circulación de los paradigmas y las políticas urbanas}

\section{Reproducción de prácticas latinoamericanas}

En América Latina hay diversos sistemas de comunicación que históricamente han permitido la difusión de políticas públicas y de experiencias urbanas heroicas (como las de la población organizada de bajos ingresos que ha construido su vivienda, su ciudad y su barrio), que han facilitado a las ciudades, sus ciudadanos y gobiernos confrontar con dignidad y eficacia diversos problemas y 
desafíos urbanos. La difusión de experiencias urbanas desarrolladas en otros contextos geográficos y en otros momentos históricos son condiciones básicas para la innovación urbana y el aprendizaje mutuo. Así por ejemplo:

- El movimiento cooperativista uruguayo de vivienda por ayuda mutua nutrió en la década de $1970^{2}$ una serie de experiencias habitacionales en la Ciudad de México ${ }^{3}$ y otras ciudades latinoamericanas. En el siglo XXI esta experiencia ha sido retomada y renovada en las áreas urbanas centrales de Buenos Aires por el Movimiento de Ocupantes e Inquilinos y en San Salvador por la Fundación Salvadoreña de Vivienda Mínima, Fundasal.

- El sistema de transporte colectivo rápido Metrobús, en un carril confinado, ideado en Curitiba, se reprodujo en múltiples ciudades latinoamericanas, entre ellas, Quito (1995), Ciudad de México (2005), Buenos Aires (2011), etc.

- Los programas de mejoramiento barrial de Suramérica, impulsados desde la década de 1980, se han expandido a México y Centroamérica en el siglo XXI.

- El modelo de los presupuestos participativos brasileiros ha sido adoptado, mal copiado o enriquecido en múltiples ayuntamientos latinoamericanos.

- La política de vivienda social chilena, construida por el sector privado con los fondos sociales, se expandió de manera colosal, salvaje y abusiva en México, y amenaza con hacerlo en otros países de la región.

Algunas de éstas políticas, programas e instrumentos urbanos han trascendido la geografía latinoamericana, para ser adaptados en algunas ciudades de Europa, como es el caso de los Presupuestos Participativos y los Programas de Mejoramiento Barrial adoptados en Cataluña.

\section{Reproducción de prácticas foráneas en América Latina}

Nuestra región históricamente ha estado vinculada e interesada en los avances urbanísticos, sociales, tecnológicos, etcétera, realizados en Europa desde el siglo XIX, y en Norteamérica después de la II Guerra Mundial. Arturo Almandoz (2002) en su bellísimo libro sobre la planeación urbana de las principales capitales de la región, realizada entre 1850 y 1950, da cuenta de la transferencia, adopción, adaptación, transformación y enriquecimiento de ideas urbanísticas foráneas. En el tránsito del siglo XIX al XX se trataba de un selectivo préstamo de ideas urbanísticas europeas, realizado por pequeños grupos sociales (elites, académicos, gobernantes) que buscaban: una identidad cosmopolita, modernizarse o seguir "perteneciendo" (en términos culturales) a Europa.

Tal vez una razón de mayor fondo era la búsqueda de alternativas e instrumentos para confrontar los emergentes problemas urbanos (congestión, hacinamiento, salud pública, etcétera) derivados del desarrollo urbano impulsado por la incorporación de las economías locales al mercado internacional, la inversión capitalista local y extranjera, la irrupción de nuevos sistemas de transporte colectivo (primero tranvías y trenes y después autobuses), el crecimiento de la población (debido a la inmigración del campo) y la expansión urbana. Al mismo tiempo que se 
buscaba higienizar la ciudad y permitir la fluidez de los nuevos transportes, se pretendía modernizarla y embellecerla. Así, en el último cuarto del siglo XIX y la primera mitad del siglo $X X$ destacadas figuras del urbanismo europeo (Forestier, Agache, Bouvard, Le Corbusier, y después Sert) fueron invitados a trabajar o a aportar ideas para la modernización de varias ciudades latinoamericanas (Hardoy, 1995). Queiroz y Pechman (1996) analizan la presencia de urbanistas franceses en Brasil. También pueden mencionarse las iniciativas de algunos arquitectos, como Carlos Contreras (Ríos, 2008) y Karl Brunner (Hoffer, 2003), que entre las décadas de 1920 y 1940 intentaban introducir en los países latinoamericanos la planificación urbana practicada en ciudades de los países más desarrollados.

Asimismo, otros urbanistas foráneos, residentes temporales o permanentes de nuestra región, han aportado sus conocimientos y experiencia en la búsqueda de nuevos rumbos para la planificación urbana, la modernización de las ciudades y la solución de los problemas urbanos locales; y han realizado ricas aportaciones teóricas. Por citar a algunos de ellos podemos mencionar a Gilbert (1997), Gormsen (1981 y 1989), y Bähr y Mertins (1995).

Actualmente las "novedades" urbanísticas europeas y anglosajonas continúan siendo una referencia en América Latina. Se trata de una relación dialéctica y asimétrica. Por un lado, algunos arquitectos, urbanistas y gobernantes latinoamericanos buscan ávida y acríticamente emular las "modas" urbanísticas del Ilamado "primer mundo"; en tanto que otros latinoamericanos lo hacen de manera crítica. Por otro lado, algunos consultores, gobiernos y casas editoriales difunden masiva y estratégicamente las "novedades" urbanísticas de sus ciudades, con el propósito de vender servicios, programas, políticas y proyectos a las urbes del llamado "tercer mundo". Aquí, para hacer negocios, se trata de transferir políticas, sistemas de planificación estratégica y formas de gestión que han demostrado su "éxito" en las ciudades del "primer mundo". Esta segunda actitud coincide con la de los organismos internacionales de financiamiento, como el Banco Mundial y en nuestra región el Banco Interamericano de Desarrollo, instituciones que promueven un "menú" de "recetas probadas" en distintos contextos urbanos para confrontar "con éxito" algunos problemas y desafíos urbanos. Estas instituciones de financiamiento fueron actores clave en el período de la guerra fría y tuvieron un papel importantísimo como promotores del "desarrollismo" en las décadas de 1950 a 1970, y en la década de 1990 participaron activamente en los procesos de reformas estructurales (políticas y económicas) en América Latina.

Algunos ejemplos dan cuenta de la diversidad de prácticas foráneas reproducidas en América Latina, lo que abarca desde la producción de planes estratégicos, megaproyectos hasta la adaptación de algunos instrumentos urbanísticos.

\section{El modelo Barcelona for export}

El proceso de transformación urbana de Barcelona con motivo de las olimpiadas de 1992, realizado entre 1980 y 1995 fue reconocido como un modelo exitoso por diversas agencias internacionales como 
el Programa de Gestión Urbana, el Centro Hábitat de Naciones Unidas, la cooperación alemana GTZ, el Programa de Naciones Unidas para el Desarrollo, etcétera. Este "modelo" ha sido ampliamente promovido y difundido en muchas ciudades. En este contexto, Borja y Castells (1997) reconocen el papel de los consultores catalanes en el Plan para Puerto Madero de 1990 en Buenos Aires, ${ }^{4}$ y en la elaboración de los Planes Estratégicos de Río de Janeiro de 1995 y de Bogotá del año 2000, en los que el mismo Jordi Borja fue consultor. Se trata de una gran campaña realizada por algunos consultores catalanes para conquistar ávidos mercados (es decir, ciudades y ayuntamientos) interesados en emular el éxito vendido por Barcelona. El libro de Puig (2009), Marca Ciudad, más que académico desde la dedicatoria parece un folleto que vende la adaptación del modelo Barcelona "para las ciudades Latinoamericanas que están rediseñando su futuro".

Golda (2007) da cuenta de la aplicación de los principios urbanísticos barceloneses en el Plan del Centro de Lima de 1989 con todo "el dogma de la multifuncionalidad del espacio público" en el que el Parque de la Exposición (cuyo origen se remonta a 1872) se transformó en Parque de la Cultura. En este caso el promotor del plan fue un arquitecto local que estudió y trabajó en la capital catalana.

Por su parte, un híper crítico local de ese "modelo" (Delgado, 2007) dice rabiosamente que se trata de la "venta de mentiras": una ciudad habitada que ha sido "ordenada", maquillada, empaquetada y vendida como si fuera una mercancía, una top model. Para él, se vende una Barcelona aséptica, bien portada, gentrificada y parquetematizada, una ciudad donde los pobres, inmigrantes ilegales y marginales han pagado muy altamente el costo en la producción de un "modelo" socialmente excluyente.

\section{El Banco Interamericano de Desarrollo (BID)}

El BID es una institución que promueve el otorgamiento de créditos para la realización de diversos programas y políticas urbanas para confrontar diversos problemas en las ciudades y promover el desarrollo: construcción de infraestructura y vivienda, recuperación de centros históricos, mejoramiento de barrios, etc. El otorgamiento de los préstamos del BID está condicionado a la adopción de formas de gestión y administración de los créditos adquiridos por los gobiernos nacionales y locales, para facilitar el funcionamiento del libre mercado. Así por ejemplo, en la década de 1970 el BID promovía el turismo en las áreas patrimoniales (zonas arqueológicas de Perú y centros históricos de Cuzco y Panamá), mientras que desde la década de 1990 el BID, igual que el Banco Mundial, reconoce al patrimonio cultural como un capital económico capaz de generar riqueza. En el ámbito de la recuperación del patrimonio cultural esto se traduce en el impulso a la participación del sector privado en el financiamiento, rescate y usufructúo del patrimonio cultural, lo que abarca la (des)regulación urbana; la generación de condiciones idóneas para la operación del mercado inmobiliario; y el retorno de la buena clientela (ver Rojas y De Moura, 1999). Un caso paradigmático fue el préstamo otorgado en 1994 para la recuperación del Centro Histórico de Quito, ciudad que tuvo que crear 
una institución público privada, la Empresa de Desarrollo del Centro Histórico, para operar ese crédito e incorporar al sector privado en la recuperación y aprovechamiento de la herencia edificada.

\section{Algunos instrumentos para el desarrollo urbano}

En materia de instrumentos urbanísticos destacan, por ejemplo, las Zonas Especiales de Desarrollo Controlado (Zedec), actuales Programas Parciales de Desarrollo Urbano, de la Ciudad de México, inspiradas en las Zones de Amenagement Controllé (ZAC o Zonas de Gestión Controladas) de Francia. Mientras que los Sistemas de Transferencia de Potencialidad del Desarrollo Urbano o del Potencial de la Capacidad Construible aplicados en las Ciudades de México, Río de Janeiro y Buenos Aires, están inspirados o son adaptaciones de los Sistemas de Desarrollo Transferibles de las ciudades estadounidenses (ver Rojas et al., 2004, p. 224).

Centro periferia, original y copia, importación y exportación de políticas urbanas

En el debate sobre la forma en que circulan las ideas, los paradigmas y las políticas urbanas aún permanecen algunas visiones anglo y eurocentristas, en donde igual que en las visiones dependentistas, prevalece la idea de que las innovaciones urbanas provienen del corazón de occidente (el primer mundo, las ciudades globales, los países más desarrollados) y desde allí se diseminan al resto del mundo. Esta idea es a menudo constatada al evidenciarse que varios políticos, urbanistas, planificadores urbanos y arquitectos de la región, con gusto importan sistemas de planificación y ordenamiento territorial, proyectos, políticas y modelos urbanos foráneos (fundamentalmente europeos y anglosajones) para intentar confrontar problemas urbanos locales 0 desarrollar estrategias de competitividad urbana; mientras que los académicos y otros estudiosos de los temas urbanos con gusto adoptan teorías urbanas foráneas para explicar los procesos urbanos locales. ${ }^{5}$

Sin embargo, frente a estas visiones simplistas que reducen la circulación de las ideas, los paradigmas y las políticas urbanas a esquemáticos procesos lineales (centroperiferia, original-copia) se ha avanzado y actualmente se reconoce que se trata de procesos más complejos (Jajamovich, 2013), bien lejos de las visiones eurocentristas ${ }^{6}$ y reduccionistas:

- América Latina ha mantenido un diálogo respetuoso y crítico, así como un interés constante con las aportaciones teóricas urbanas provenientes de los países hegemónicos (con sus poderosas cadenas editoriales que inundan nuestros mercados de libros con traducciones al español y al portugués).

- La circulación de las ideas se ha acelerado y multiplicado en un mundo globalizado.

- Muchos colegas "foráneos" que se han avecindado y radicado temporal o permanentemente en América Latina, han realizado grandes aportaciones prácticas y teóricas para la comprensión de los procesos urbanos locales. 
Conviene citar aquí dos críticas a las visiones eurocentristas:

- Robinson (2009) señala que la modernidad más que occidental es cosmopolita, y que la idea de la invención, apropiación o copia es una actitud o toma de posición frente al mundo. Ella pone de ejemplo dos ciudades, Nueva York y Rio de Janeiro, que a fines del siglo XIX y principios del XX adaptaban con gusto los modelos urbanísticos y arquitectónicos franceses: la primera se asume como ciudad moderna y cosmopolita, y olvidó que su cosmopolitismo urbanístico es una apropiación, copia e imitación de otras culturas; mientras que en la segunda, desde la década de 1920 se mantiene la idea de que la modernidad es foránea y es una copia que no ha podido invisibilizar la pobreza urbana.

- Este mismo debate se repite en un libro reciente sobre el nuevo urbanismo colonizador. Atkinson y Bridge (2005) presentan la idea de establecer una sede del Museo Guggenheim en Rio de Janeiro, a principios del siglo XXI, como una clara evidencia del urbanismo colonizador. Sin embargo, en ese mismo libro la sede de ese museo en Bilbao aparece como una marca y un modelo exitoso de renovación urbana ¿Porqué en Rio una sede del Guggenheim es una evidencia del urbanismo colonizador y en Bilbao no?

Es evidente que las teorías surgidas en países foráneos, particularmente los anglosajones, han tenido una influencia en la agenda de investigación de la región (el postmodernismo, la ciudad global, etc.). Sin embargo, rechazamos la idea que coloca a los investigadores latinoamericanos como actores pasivos que adoptan paradigmas en boga. Muchos latinoamericanos hemos estudiado en países del llamado primer mundo y a diferencia de muchos colegas monolingües (sobre todo los anglosajones) leemos y nos mantenemos actualizados sobre lo que se escribe en otras lenguas sobre el mundo urbano (hasta traducimos y reseñamos trabajos escritos en idiomas foráneos), pues tenemos un interés en el conocimiento universal y de ninguna manera renunciamos a las aportaciones extranjeras. Sin embargo, retomamos críticamente lo que consideramos que aporta para explicar nuestras y otras realidades urbanas.

Asimismo, vivimos en un mundo globalizado en el que (así sea de forma asimétrica) tenemos acceso a un importante cúmulo de conocimiento científico, e interactuamos directa e indirectamente con colegas, expertos y profesionistas de los países del norte y/o del sur que van y vienen a estudiar o son enviados a cooperar y trabajar en el sur (y en menor medida en sentido contrario). Estas relaciones (cada quien con su formación profesional, académica, así como con sus paradigmas, visiones urbanas e intereses profesionales y económicos), que se realizan en el marco de profundas desigualdades económicas entre las partes participantes de estos intercambios, contribuyen al enriquecimiento científico y al avance de las ciencias sociales en materia de los estudios urbanos.

Por ello, en nuestro quehacer profesional promovemos la difusión crítica de las aportaciones teóricas foráneas y de las buenas políticas locales y foráneas que adaptadas a las condiciones locales pueden coadyuvar a la solución de problemas 
específicos. Sin embargo, somos críticos de las llamadas "buenas prácticas", especialmente cuando quienes las promueven son organismos internacionales legitimadores del modelo neoliberal.

\section{Políticas, programas y acciones urbanas ¿urbanismo a la carta?}

La repetición del mismo tipo de programas y políticas urbanas y la difusión "exitosa" de algunas experiencias urbanísticas en varias ciudades latinoamericanas gobernadas por partidos políticos con las más diversas orientaciones políticas, conducen a plantear la hipótesis de que existe un "Urbanismo a la Carta" para confrontar diversos problemas y desafíos urbanos, que se oferta a los gobiernos locales y nacionales por parte de consultores, bancos de desarrollo, agencias de cooperación internacional, la Organización de Naciones Unidas y otras instituciones internacionales. Este "Urbanismo a la Carta" presenta, cual menú de restaurante, una variedad de entradas, ensaladas, platos fuertes y postres ad hoc para distintos gustos y precios, lo que abarca la "cocina" típica, regional e internacional.

Urbanismo a la Carta, Menú

\begin{tabular}{|c|c|}
\hline $\begin{array}{c}\text { Acciones puntuales } \\
\text { De bajo costo y con una gran visibilidad mediática }\end{array}$ & $\begin{array}{l}\text { Programas urbanos diversos } \\
\text { De bajo costo que no necesariamente implican } \\
\text { la realización de obra pública }\end{array}$ \\
\hline $\begin{array}{l}\text { Programación de eventos culturales en espacios públicos. } \\
\text { Introducción de políticas y programas de seguridad pública. } \\
\text { Promoción del turismo urbano. } \\
\text { Instalación de playas urbanas en el verano. } \\
\text { Introducción de carriles confinados para el uso de bicicletas. }\end{array}$ & $\begin{array}{l}\text { Formulación de planes estratégicos. } \\
\text { Otorgamiento focalizado de becas para población vulnerable. } \\
\text { Aplicación del ejercicio de los presupuestos participativos. } \\
\text { Reubicación del comercio informal. } \\
\text { Introducción de parquímetros (para ordenar el tráfico y } \\
\text { desplazar a los "cuida" autos). } \\
\text { Remozamiento y recuperación del espacio público. Introducción } \\
\text { de eco bicicletas como sistema de transporte público. }\end{array}$ \\
\hline Proyectos y megaproyectos urbanos & Estrategias de marketing urbano \\
\hline $\begin{array}{l}\text { Realización de Megaproyectos: recuperación de frentes } \\
\text { fluviales, reconversión de zonas fabriles, construcción de } \\
\text { proyectos ícono, etc. } \\
\text { Recuperación de centros y barrios históricos. } \\
\text { Introducción del Sistema de Metrobús en un carril confinado. } \\
\text { Programa focalizado de mejoramiento de barrios. } \\
\text { Programas de mejoramiento de vivienda popular con } \\
\text { microcréditos. }\end{array}$ & $\begin{array}{l}\text { Campañas de marketing urbano: Ciudad Verde, Ciudad global, } \\
\text { Ciudad compacta, Ciudad sustentable, equitativa y competitiva } \\
\text { / Ciudad para todos / Ciudad de la esperanza / Primero los } \\
\text { pobres (porque siguen los ricos). } \\
\text { Realización y participación en conferencias internacionales } \\
\text { para presentar los "logros" de una "gestión exitosa". } \\
\text { Negociar en foros internacionales para ser la sede de algo: } \\
\text { Capital Cultural, Encuentro internacional de Alcaldes, etc. }\end{array}$ \\
\hline
\end{tabular}

Nota: Los "platillos" del menú pueden cambiar de lugar y ser ofrecidos en paquete (menú completo) o en partes. Un pedido puede no tener entradas o postre. Así por ejemplo el comensal (ayuntamiento, gobernante, inversionista) puede considerar la elaboración de un Plan Estratégico o una campaña de marketing como "el plato fuerte" de su gestión o inversión. 
Los chefs o cocineros de este "Urbanismo a la Carta" son destacados académicos y consultores foráneos, los organismos financieros internacionales (en nuestra región el BID), las agencias de cooperación internacional y también los profesionistas locales en nuestro papel de académicos, consultores o funcionarios públicos.

Por cuestiones de espacio, en este artículo desarrollamos sólo algunas de las recetas de este "Urbanismo a la Carta": el sistema de transporte en bicicleta, las políticas sobre el espacio público y los programas de mejoramiento de barrios. Las razones de esta elección responden al énfasis actual de la política urbana en la Ciudad de México, en donde este conjunto de políticas públicas se presentan no sólo como políticas innovadoras sino como una invención "local". Sin embargo, como se puede comprobar en este artículo, se trata de la recreación de políticas y programas urbanos que han surgido antes o de manera casi simultánea en otras ciudades latinoamericanas. Asimismo, antes de presentar algunos programas y políticas urbanas considero necesario presentar algunos datos de las tres ciudades objeto de estudio para contextualizar las acciones desarrolladas en ellas.

\section{Algunos datos sobre Buenos Aires, Ciudad de México y Quito}

Las tres ciudades han sido sede del poder político desde la época de la colonia, son la capital de su respectivo país desde la independencia y tienen una dimensión metropolitana, pero son muy diferentes en dimensión física, demográfica, formas de gobierno, origen, función económica, historia, etcétera. Así por ejemplo, en términos de población, la zona metropolitana de la Ciudad de México es una vez y media mayor que la zona metropolitana de Buenos Aires y casi nueve veces mayor que el Distrito Metropolitano de Quito. Mientras que sólo la Ciudad de México es tres veces mayor que Buenos Aires Capital Federal y 5.4 veces mayor que la ciudad de Quito.

Población y Vivienda 2010

\begin{tabular}{|l|r|c|r|r|r|r|}
\hline & \multicolumn{2}{|c|}{ Buenos Aires } & \multicolumn{2}{c|}{ Ciudad de México } & \multicolumn{2}{c|}{ Quito } \\
\cline { 2 - 7 } & Metrópoli & Ciudad & Metrópoli & Ciudad & Metrópoli & Ciudad \\
\hline Población & $12,801,364$ & $2,891,082$ & $20,019,381$ & $8,851,080$ & $2,239,191$ & $1,619,146$ \\
Vivienda & $4,230,636$ & $1,082,998$ & $5,827,109$ & $2,453,770$ & 763,719 & 550,265 \\
\hline
\end{tabular}

Fuentes: Elaboración propia con base en datos del Inec (2010); Inegi (2010). 
Sin embargo, las tres ciudades y los tres países presentan problemáticas económicas, políticas públicas y turbulencias políticas semejantes o coincidentes. Así por ejemplo: 1) En el marco de reformas políticas en 1996 y 1997 las ciudades capital de Argentina y México, respectivamente, eligieron democráticamente a sus gobiernos locales por primera vez en la historia; 2) La economía argentina estuvo dolarizada durante un período de 10 años y la ecuatoriana se encuentra dolarizada desde 2001, en ambos países un mismo personaje fungió, en diversos momentos, como secretario de economía; 3) A pesar de procesos locales de desindustrialización, las tres ciudades contribuyen de manera importante a la economía nacional. En el año 2000 Buenos Aires aportaba el $25 \%$ del producto interno bruto nacional y la Ciudad de México el $22.3 \%$, mientras que Quito concentraba el $30 \%$ de la industria nacional; 4) Los tres países han padecido fuertes crisis económicas y grandes cambios políticos desde 1990:

- La economía mexicana sufrió un crack en diciembre de 1994 y su moneda se devaluó fuertemente en el trascurso de 1995; la economía del Ecuador tuvo una fuerte crisis en 1999 y en el 2001 se dolarizó; mientras que la economía argentina tuvo su crack entre 2001 y 2002 y se desdolarizó ese último año.

- Constantes crisis políticas, económicas y financieras, generadas por la introducción de políticas de ajuste de la economía y casos de corrupción, han ocasionado la destitución de varios presidentes en Ecuador (Bucaram en 1997, Mahuad en 2000 y Gutiérrez en 2005) y Argentina (De la Rúa en 2001, aquí además transitaron varios presidentes interinos).
Introducción de sistemas de transporte en bicicletas

Este apartado está basado en trabajo de campo realizado en febrero de 2013 (Quito), mayo de 2013 (Buenos Aires) y durante agosto - octubre de 2013 (Ciudad de México), así como en información obtenida en las siguientes páginas de internet, visitadas en octubre de 2013: Gobierno Autónomo de la Ciudad de Buenos Aires www. ecobicibuenosaires.gob.ar; Gobierno de la Ciudad de México www.ecobici.df.gob.mx; Gobierno de Quito www.biciq.gob.ec/web.

Buenos Aires, la Ciudad de México y Quito han introducido recientemente el sistema de transporte público en bicicletas. En las dos primeras esto ocurrió en los meses de marzo y febrero de 2010 respectivamente, y en Quito en julio de 2010. En las tres ciudades el sistema incluye el confinamiento de algunos carriles para la circulación exclusiva en bicicleta, un sistema de disposición de bicicletas en préstamo en Buenos Aires, y en alquiler Quito (25 dólares anuales) y Ciudad de México (con costos desde 400 a 90 pesos mexicanos si es por un año o por un día respectivamente).

El sistema más grande es ofrecido en la Ciudad de México con 275 estaciones, cuatro mil bicicletas y 87 mil usuarios; seguido de Buenos Aires con 28 estaciones, 750 bicicletas y 70 mil usuarios registrados. Mientras que en Quito el sistema tenía 25 estaciones y 425 bicicletas. Buenos Aires pretende incrementar el sistema hasta llegar a 3 mil bicicletas, 200 estaciones y automatizar el servicio para funcionar las 24 horas del día. 
Curiosamente el Gobierno de la Ciudad de México, que se autodesigna de izquierda, es el único que ha concesionado el servicio EcoBici. Aquí el sistema es operado por Clear Channel a través de su división Smartbike (una firma que opera en Oslo, Barcelona, Estocolmo, Verona y otras ciudades) en conjunto con una de las empresas de Carlos Slim (el inversionista más rico del país) que puede cobrar el alquiler de una Ecobici a través del sistema telefónico Telmex. En cambio, en Buenos Aires Mauricio Macri no consiguió concesionar el sistema Mejor en Bici o Ecobici por oposición social y en el parlamento local.

Llama la atención que el sistema funciona en la parte bonita o moderna de las tres ciudades (centro de las ciudades, distritos de negocios, centros y barrios históricos recuperados), al margen de los barrios populares y bien lejos de los barrios periféricos, donde vive la mayor cantidad de población. Si bien es cierto que el discurso en la promoción de este sistema de transporte público, es justamente contribuir a la descongestión del tráfico en las áreas urbanas centrales, curiosamente estos sistemas no promuevan una cobertura en los barrios populares céntricos y en las periferias urbanas, donde centenas de miles de personas realizan desplazamientos locales para ir a la escuela, de abasto y de compras. Tal vez estos programas de transporte no se realizan en los barrios pobres porque se teme que la población no pague por el alquiler de la bicicleta o se la robe para venderla o para su consumo personal.

También es revelador que en los argumentos oficiales en favor de este sistema aparezca de manera protagónica una comparación con las metrópolis del primer mundo. Así:

- Mauricio Macri señala que éste es un programa "en línea con las tendencias mundiales" y con las grandes capitales como "París, Nueva York, Barcelona y Bogotá" (Fuente: GCABA Boletín del 22/6/2012).

- En la inauguración de EcoBici en la Ciudad de México, en febrero de 2010, el alcalde Marcelo Ebrard decía que éste sistema "nos pone al mismo nivel que ciudades como Barcelona, París y Washington" (Fuente: El Universal 16/2/2010).

\section{Políticas sobre el espacio público}

El espacio público, como concepto, tiene múltiples significados y dimensiones: política, física, urbana y otros más. Algunas visiones reducen este concepto al ámbito de la ideología dominante o a los espacios urbanos abiertos. Nosotros reivindicamos una definición más amplia del concepto que puede inscribirse en el ámbito de la filosofía política. En una versión sintética, Rabotnikof (2010) define el espacio público por tres criterios y principios básicos: 1) lo que es de utilidad o de interés común para todos (una comunidad o colectivo), 2) lo que se hace y desarrolla a la luz del día, lo manifiesto y lo ostensible, y 3) lo que es de uso común, lo que está abierto y es accesible para todos. Esta definición amplia abarca la política, la economía, la educación, la ciudad, la salud, los medios de comunicación, el medio ambiente, etcétera. Se trata de una definición de principios que no necesariamente coincide con el espacio público que tenemos o hemos tenido. En este sentido se trata (como el 
Derecho a la Ciudad) de un espacio público a (re)conquistar o a construir.

Para Merino (2010) el espacio público se corrompe, cuando su naturaleza se convierte en privada, se oculta lo que debe saberse, los atributos de inclusión y accesibilidad se transforman en excluyentes, lo abierto se cierra, los recursos públicos se utilizan como si fueran privados, la información pública se usa de manera privada, se oculta el origen y destino de los recursos públicos, se cierran las calles y plazas en beneficio privado, y el gasto social y la política pública se tornan excluyentes e inaccesibles. ${ }^{9}$

Para Borja (2003) el espacio público no se reduce a un suelo con un uso y función especializados, sino que el espacio público es la ciudad: un lugar abierto y accesible para todos, un sitio significante en el que confluyen todo tipo de flujos, y un lugar político de representación y de expresión colectiva de la sociedad. Por su parte, el espacio público en su dimensión física es un espacio funcionalmente polivalente, que articula todo con todo, ordena la urbe, facilita el encuentro, el intercambio, la movilidad y la accesibilidad de los recursos urbanos, así como la permanencia de las personas.

Para Delgado (2011) el espacio público es un concepto que tiene una yuxtaposición de concepciones e interpretaciones que abarcan: los lugares de libre acceso, los ámbitos para los vínculos sociales, los espacios de relación con el poder, los sitios del público y para las relaciones en público, etcétera. La presencia del espacio público ratificaría la democracia y promovería el encuentro y las reuniones sociales. Sin embargo, el espacio público no es por naturaleza un lugar democrático para un ciudadano abstracto e ideal, ni tampoco borra las desigualdades sociales. Al contrario, actualmente el espacio público es una ideología que sirve a las construcciones y a los negocios que rodea y se llena de modo adecuado para los objetivos de los inversionistas y los gobiernos. Como si hablara de nuestro "Urbanismo a la carta", Delgado señala que las intervenciones en el espacio público son una "guarnición de las grandes operaciones inmobiliarias" (algo así como una orden de papas fritas para un buen bife).

Delgado (2011) afirma que el espacio público es un concepto que recientemente se puso de moda, ${ }^{10}$ a partir de los grandes megaproyectos de reconversión urbana. Se trata de una ideología que sirve para la reapropiación capitalista de la ciudad, y que bajo el argumento del paraíso de la ciudadanía (cortés, consciente y bien portada) excluye y desplaza los comportamientos inapropiados de las clases bajas (vendedores ambulantes, indigentes, inmigrantes, prostitutas, etc.). Justo por ello, las legislaciones y normativas cívicas a nombre de un espacio para todos pretenden ordenar y controlar el espacio público, y excluir los malos comportamientos. Se trata de ordenanzas del tipo de la cero tolerancia que intentan construir un estado de excepción y excluir u ocultar la pobreza.

- Aquí podemos citar como ejemplo, que el Gobierno de "izquierda" de la Ciudad de México, comandado por Andrés Manuel López Obrador, en 2002 contrató al ex alcalde republicano de Nueva York, Rudolf Guliani, para que lo asesorara en materia de la seguridad pública en dos territorios emblemáticos en proceso de "recuperación": 
el Centro Histórico y el Paseo de la Reforma. En el marco de las recomendaciones de Guliani, la mayoría de "izquierda" en el parlamento local aprobó en 2004 la Ley de Cultura Cívica, que otorga instrumentos jurídicos al gobierno local para combatir el comercio y los servicios informales, el grafiti y los sospechosos.

\section{Autoridades y programas de recuperación, construcción y ampliación \\ del "espacio público"}

En las tres ciudades objeto de estudio ha emergido con fuerza, en la última década, el tema del Espacio Público, al grado que los tres gobiernos locales han creado instituciones específicas para ello. Lo que no omite que antes de ello se hayan realizado proyectos de mejoramiento del espacio público en barrios y centros históricos, bulevares (como la recuperación del Paseo de la Reforma en la Ciudad de México 2000-2006 y de la Avenida de Mayo en Buenos Aires 1991-1993) y de otras calles simbólicas.

En efecto, en 2008 se creó la Autoridad del Espacio Público en la Ciudad de México (AEP, 2012), mientras que la Gerencia de Espacio Público en Quito dejó de depender de la Dirección Metropolitana de Territorio y Vivienda para pasar a la Empresa Pública Metropolitana de Movilidad y Obras Públicas (Salazar, 2011). Por su parte, en 2007 se fusionaron dos ministerios en Buenos Aires para formar el Ministerio de Ambiente y Espacio Público (GCABA, 2013). Las tres dependencias públicas tienen funciones similares, son órganos de gestión y realización de obra pública como la mejora y sustitución de mobiliario urbano y alumbrado público; la arborización y cuidado o creación de áreas verdes y plazas con juegos de agua, aparatos de ejercicios, etc.; o el cuidado y pavimentación de calles. En Quito esa dependencia se ocupa de la nomenclatura y la publicidad; y en Buenos Aires de la basura. Destacan dos funciones en dos ciudades:

- la Autoridad del Espacio Público, en la Ciudad de México, tiene como función la mejora del espacio pública para crear las condiciones económicas que incentiven la inversión productiva y fomenten la creación de empleo (AEP, 2012).

- en Buenos Aires destaca el eslogan de Ciudad Verde y el impulso -desde 2008- al Plan "Guardianes de la plaza" consistente en un cuerpo de "Intendentes de plaza" que difunde las normas básicas sobre el buen uso del espacio público, disuade las actividades prohibidas y denuncia los delitos. Hay 380 guardianes (en tres turnos) para 88 Espacios Verdes en la capital porteña (GCABA, 2013).

Entre los proyectos emblemáticos se pueden mencionar:

- en Buenos Aires: 1) la Remodelación del Microcentro, con la peatonalización de varias calles y la sustitución de redes de infraestructura; 2) el Remozamiento de Palermo Viejo, y 3) el remozamiento de una parte (27 de 130 hectáreas) del Parque Indoamericano inaugurado en diciembre de 2011.

- en la Ciudad de México la Autoridad del Espacio Público en su corta vida ha realizado 15 proyectos, los más emblemáticos son: 1) el remozamiento de la Plaza, Monumento y Museo de la Revolución, 2) el conjunto de la Plaza Garibaldi (con el Museo del Tequila, el Mercado San Camilito y la Academia del Mariachi); el Corredor peatonal Madero, la 
Azotea Verde y el remozamiento de la Av. Juárez y la Alameda Central, la Av. Pino Suarez y la Plaza Tlaxcoaque. Todos ellos en el centro histórico.

- en Quito: 1) la construcción del Bulevar Avenida Naciones Unidas con la ampliación y mejoramiento del espacio público, lo que recupera el prestigio de ese sector; 2) la creación de la Plaza Cultural Quitumbe en el Sur de la Ciudad; 3) el remozamiento de la Av. Napo, en la zona centro sur; y 4) el mejoramiento del espacio público y la infraestructura de La Mariscal Sucre, un barrio turístico y de servicios.

- en Buenos Aires y la Ciudad de México las autoridades sobre el espacio público también rescatan o mejoran los llamados "Bajo puente" o "Pasos bajo nivel" con iluminación, cámaras de video seguridad y pintura antigrafiti.

- en Quito esa autoridad es la encargada de la administración de los 9,762 estacionamientos concesionados o privados del área central, mientras que en la Ciudad de México la autoridad es la responsable de la instalación de parquímetros en algunas colonias centrales de clase media y alta.

En las tres ciudades se promueve la realización de exposiciones y eventos culturales al aire libre en los espacios públicos remozados, creados o ampliados (esculturas urbanas, pinturas, murales, etc.). Igualmente en Buenos Aires se realizó en 2011 el Encuentro Internacional Street Art y en la Ciudad de México ese mismo año se realizó una actividad similar a la que acudieron los más famosos grafiteros, stencileros y muralistas del mundo. Es curioso como el concepto de grafiti tiende a ser, o ha sido, sustituido por el de murales, esténciles, Street Art y Urban Art.
Asimismo, es evidente que el remozamiento o creación de espacios públicos privilegia selectas áreas urbanas centrales, sobre todo en la Ciudad de México, en donde prácticamente toda la intervención pública se concentra en la parte "bonita" del centro de la ciudad, donde viven o consumen las clases medias y altas. Con ello, se fortalece el patrón de segregación funcional y social de la ciudad. En cambio, en Quito y Buenos Aires hay obras simbólicas y puntuales que se han realizado en zonas populares como el parque Indoamericano (así sea para ocupar políticamente un territorio invadido en $\mathbf{2 0 1 0}$ por personas sin techo) y la Plaza Quitumbe respectivamente.

Un último aspecto que me interesa destacar es el discurso de los alcaldes y gobiernos locales sobre el espacio público, así como los eslóganes que usan y responden a campañas de marketing:

- Macri habla de que "El espacio público (...) es el lugar más democrático y el que más necesitan los que menos tienen" y señala que "El Parque [Indoamericano] recupera su razón de ser: un espacio público de todos y para todos". Fuente: GCABA: Comunicado del 13/2/2013.

- El anterior alcalde de la Ciudad de México, Ebrard, decía que "El espacio público permite reducir la segregación que produce la economía", pero que en el centro histórico "no se permitirá que haya vendedores ambulantes, porque el espacio público es lo que nos hace ciudadanos y nadie tiene derecho a apropiárselo por ningún motivo". Fuente: $L a$ Jornada 19/7/2012.

- Por su parte, el gobierno local de Quito dice que genera y recupera espacios públicos en la 
ciudad (aunque además del megaproyecto de la Avenida Naciones Unidas sólo ha emprendido dos más, de menor dimensión e inversión) para disminuir los desequilibrios urbanos acercando los equipamientos y servicios a la ciudadanía.

Marketing urbano, eslóganes de gobierno: El Gobierno de Macri agrupa, bajo el eslogan de Ciudad Verde, varios de los programas públicos aquí referidos, mientras que en toda la publicidad y difusión de los programas y políticas de gobierno usa En todo estás Vos. Ambos eslóganes son muy parecidos a los que el actual y el anterior gobierno de la Ciudad de México usaban y usan:

- Marcelo Ebrard (2006-2012) usaba un anodino eslogan de gobierno "Ciudad en Movimiento", tal vez en abierta oposición al de su antecesor López Obrador (2000-2006) que usaban "La ciudad de la esperanza", pero en cambio su eslogan de desarrollo urbano era "competitivo, equitativo y sustentable".

- por su parte, el actual alcalde, Miguel Ángel Mancera (2012-2018), usa como eslogan de gobierno "Decidiendo juntos" y para el desarrollo urbano el de "Ciudad compacta".

Es evidente que los eslóganes de gobierno se amparan en las ideas de la atención y participación ciudadana; y los de desarrollo urbano en el tema de la sustentabilidad urbana, el urbanismo verde y el desarrollo urbano intensivo. Sin embargo, se trata de meros discursos que no resisten la mínima prueba de la participación ciudadana y del desarrollo urbano sustentable, pues a menudo bajo estos emblemas se practican políticas públicas en sentido contrario.

\section{Programas de mejoramiento de barrios}

Los programas de Mejoramiento de Barrios se remontan a las décadas de 1960 y 1970, cuando los gobiernos de diversas ciudades latinoamericanas recurrieron a la regularización de asentamientos informales y posteriormente introducían infraestructuras y otras medidas de saneamiento. Sin embargo, la versión actual de esta política focalizada se remonta a la década de 1980. El BID es un activo agente que desde la década de 1980 ha otorgado créditos para el mejoramiento barrial (hasta 2008 había financiado 37 proyectos de mejoramiento barrial en 13 países) con el propósito de garantizar la gobernabilidad urbana, evitar conflictos sociales y combatir la pobreza (Rojas, 2009).

En Argentina, Ecuador y México es evidente el papel protagónico del BID, institución que otorgó el primer crédito para este propósito a Chile en 1986 y cofinanció el emblemático programa Favela Bairro desde 1995. Esta política focalizada, en términos sociales y territoriales, pretende integrar física y socialmente los asentamientos populares a la ciudad a través de un conjunto de acciones físicas, jurídicas y sociales, entre las que destacan: la dotación de infraestructuras, la recuperación de áreas medioambientalmente degradas o no urbanizables; la regularización de la tenencia de la vivienda (esto no aplica en muchas ciudades); y la introducción de servicios sociales y comunitarios. Los programas de más reciente generación también han pretendido incorporar medidas para reducir la violencia y combatir la inseguridad pública. Asimismo, 
se ha pretendido incidir en la economía local, a través de la creación de empleos; facilitar el acceso a créditos a través de la regularización de la propiedad inmueble; y eliminar el estigma socio espacial a través del acceso a una dirección y a un barrio formal.

En Argentina el Programa de Mejoramiento Barrial (PROMEBA I) data de 1996 y una segunda fase (PROMEBA II) se realizó a partir de 2007. Este programa no ha operado en Buenos Aires, tal vez porque la dimensión de la problemática constituida por las Ilamadas Villas Miseria es insignificante comparada con la de las otras 19 provincias argentinas, o bien porque no ha habido la intención de reconocer esos asentamientos informales. En cambio, en Ecuador y México esa política se remite apenas al siglo XXI con los programas de Apoyo al Sector Vivienda (2002) y de Mejoramiento Integral de Barrios (2007); y Hábitat I (2003) y Hábitat II (2007) respectivamente. En ambos casos, los asentamientos informales están excluidos de esta política pública. En la capital mexicana ese programa nacional financiado por el BID no opera, porque un gobierno de izquierda "no trabaja" con instituciones neoliberales. Sin embargo, como se verá enseguida, no hay diferencia alguna entre las políticas de mejoramiento barrial financiadas por el BID y el Programa Comunitario de Mejoramiento Barrial que desde 2007 y desde la "izquierda" se opera en la capital mexicana.

En efecto, los barrios pobres y marginados de Quito y la Ciudad de México se deben organizar para competir por los recursos públicos para mejorar sus barrios. En la Ciudad de México en seis años se atendió a 981 barrios (la tercera parte de los 3,328 barrios que compitieron para ganar los recursos públicos) y los programas de mejoramiento de la imagen urbana abarcaban el $40 \%$ de los barrios atendidos (pintura de fachadas); la construcción o rehabilitación de espacios comunitarios alcanzaba una cuarta parte (25\%); y el mejoramiento de diversas áreas comunes y jardines constituían el resto (22\%).

Se trata de nobles pero insuficientes políticas públicas que son definidas como universalistas, pero tienen una forma de operación focalizada. Los barrios pobres deben organizarse y concursar por la obtención de fondos públicos, y los escasos recursos se reparten entre el mayor número posible de población y de barrios. En este sentido hay una serie de preguntas sin respuesta: ¿Se atiende a la población más pobre o los beneficios son capturados por los menos pobres? ¿La población más pobre tiene capacidad de organizarse y concursar un proyecto?

\section{Algunas conclusiones}

\section{Para una agenda sobre la teoría urbana en América Latina}

Este artículo ha pretendido modestamente contribuir a la apertura de una línea de investigación sobre la circulación de las políticas urbanas recientes que de manera simultánea o diacrónica se están reproduciendo en diversas ciudades de América Latina. Aunque este artículo se limita a la comparación de tres tipos de políticas urbanas que se realizan en tres ciudades, sabemos que estas políticas se reproducen, recrean, adoptan y adaptan en 
muchas ciudades de la región. Ello representa un desafío y una línea de trabajo para futuras investigaciones comparadas.

La circulación de ideas y políticas urbanas se ha intensificado merced a la propagación de las nuevas tecnologías de las telecomunicaciones y a la multiplicación de los intercambios, reales y virtuales, entre gobernantes, académicos, estudiantes y profesionistas de diversas ciudades, en múltiples escalas: sur-sur, norte-sur, sur-norte, etc. Hasta donde alcanzamos a ver la actual circulación de paradigmas y políticas urbanas ocurre en múltiples escalas y en dos circuitos de manera simultánea y yuxtapuesta:

- un circuito hegemónico y bien neoliberal, promovido por gobernantes, consultores y agencias de desarrollo internacional,

- un conjunto de redes alternativas promovido por gobernantes y académicos críticos, organismos civiles y sociales, que lejos de la doctrina neoliberal, y con una actitud universal y abierta al mundo, aprenden de experiencias foráneas (locales, regionales e internacionales) para intentar confrontar de mejor manera los desafíos urbanos del siglo XXI y las necesidades de la mayoría de nuestra población.

En el primer caso destaca el papel desempeñado por el BID que promueve la reproducción de lo que esa institución considera como "buenas prácticas" en otras ciudades y países de la región, como es el caso de los programas de mejoramiento de barrios. También destaca el papel de los consultores catalanes en la elaboración de un conjunto de planes estratégicos para distintas partes de diversas ciudades (así por ejemplo, el libro de Borja y Castells (1997), contiene varios recuadros que sintetizan la labor de estos consultores en ciudades como Bogotá, Rio de Janeiro y Buenos Aires.

En el segundo caso pueden citarse por ejemplo el papel de la Secretaría Latinoamericana de la Vivienda y el Hábitat Popular (SELVIHP), institución creada a fines del siglo XX por un conjunto de organismos sociales, que constantemente realiza intercambios en materia de experiencias autogestivas entre sus socios miembros de Brasil, Argentina, Uruguay, Chile y Paraguay.

Sobre la circulación histórica y actual de paradigmas urbanos entre América Latina y Europa y Norteamérica se han realizado varios y muy ricos trabajos (Almandoz, 2002; Golda, 2007; Hardoy, 1995; Jajamovich, 2013; Queiroz y Pechman, 1996; etc.). Sin embargo, consideramos que tenemos pendiente la realización de investigaciones que den cuenta cómo han circulado las políticas urbanas y las aportaciones teóricas latinoamericanas en la misma región. No conocemos trabajos que de manera comparativa que presenten un conjunto de programas urbanos surgidos en el sur que se han propagado en el mismo sur y en el norte, como la política de vivienda social chilena, los presupuestos participativos brasileños, el Metrocable de Medellín y el Metrobus de Curitiba.

\section{¿lzquierda y derecha? \\ ¿Neoliberal anti neoliberal?}

Este trabajo no está en condiciones de responder fehacientemente a una de las preguntas planteadas en la introducción ¿Qué es lo que hace que alcaldes y gobiernos locales con orientaciones políticas tan diferentes 
ejecuten el mismo tipo de políticas urbanas? ¿La misma visión pragmática, coincidencia política, coacción económica, una ideología dominante? Consideramos que para responder a estas preguntas tendríamos que realizar una investigación en profundidad que en lo posible abarcara la entrevista con los gobernantes, los tomadores de decisiones y los promotores de esas políticas. Dicho análisis podría abarcar algunos aspectos biográficos relevantes, como dónde estudiaron o dónde conocieron las políticas urbanas que se adoptan. No obstante, a manera de hipótesis, estamos de acuerdo con Pradilla (entrevistado por Delgadillo, 2013) cuando sostiene que las "mismas" políticas urbanas y similares megaproyectos urbanos son reproducidos en distintas ciudades latinoamericanas por gobiernos con distintas ideologías y orientaciones políticas por tres razones fundamentales: 1) después de la caída del socialismo real se ha perdido la raíz ideológica y el pensamiento neoliberal se ha convertido en un pensamiento hegemónico del cual se alimenta la mayoría de las tendencias políticas actuales, 2) la falta de conocimiento y de ideología en muchos gobernantes y políticos les impide establecer las diferencias, 3) el pragmatismo ha reemplazado el conocimiento, de igual forma que la planeación estratégico y los megaproyectos (que tienden a sustituir a la planeación urbana tradicional), se concentran en algunas partes de la ciudad y no en el todo de la ciudad.

En este artículo, a partir de este análisis sesgado y preliminar, es curioso constatar como las orientaciones políticas que se sostienen en el discurso poco tienen que ver con la praxis. Así por ejemplo:
- el gobierno de la Ciudad de México que se autodefine como de izquierda y anti neoliberal, es más neoliberal que el conservador gobierno de Buenos Aires. La Ciudad de México es la única de las tres ciudades que tiene concesionado el sistema de transporte de bicicletas y de cobro de parquímetros. En esta ciudad, a pesar del discurso universalista, igualitarista y de izquierda, el programa de mejoramiento barrial actúa de facto con las reglas de operación del BID sin deberle crédito alguno: mejoramiento de barrios a través de concursos, hacer más (mejoramientos) con menos (presupuesto), etc.

- la correlación de fuerzas políticas locales en Buenos Aires no han permitido al conservador Jefe de Gobierno privatizar el servicio de transporte público en bicicleta. Mientras que en Quito el sistema de transporte público en bicicleta es controlado y subsidiado por el gobierno local.

\section{La modernización selectiva de privilegiados territorios céntricos}

Las tres ciudades concentran la mayor cantidad de sus inversiones y proyectos emblemáticos en las partes centrales de la ciudad, donde viven y/o consumen la clase media y media altas: los centros de negocios, centros y barrios históricos. Allí, se remoza, recrea y amplía el espacio público urbano, que además 1) se llena de actividades culturales y posibilidades lúdicas para atraer a más población; y 2) es objeto de nuevas y fuertes medidas de seguridad pública para prevenir y combatir cualquier tipo de delitos. 
En estos lugares, bellos, vibrantes, higiénicos y seguros las 24 horas del día parece cumplirse la utopía de la equidad social y territorial; el derecho a una ciudad segura y saludable donde coexisten respetosamente las diferentes culturas, etnias y género (como dice la propaganda en Quito); el lugar donde se democratiza la cultura con las exposiciones y espectáculos culturales abiertos y gratuitos al aire libre; y el sitio donde se encuentran los colectivos locales, nacionales y extranjeros en sus facetas de trabajo, estudio o recreación. Sin embargo, en dichos lugares no caben las prácticas económicas de los desempleados y subempleados que representan una gran cantidad de nuestras ciudades, quienes se dedican al comercio ambulante, a los servicios informales y a otras actividades "inadecuadas" para la dignidad de dichos espacios urbanos.

La inversión pública no sólo mejora y amplía los espacios públicos en las áreas más rentables y visibles de las tres ciudades, sino que revaloriza la propiedad privada, pero dicha inversión pública no es recuperada. Además, al no realizarse este tipo de acciones públicas (ni en su dimensión física ni económica) en espacios públicos en los barrios populares ni en las periferias distantes dichas acciones contribuyen a la profundización de la segregación social y espacial de la ciudad.

- Espacios públicos en barrios en barrios populares se realizan de manera muy puntual en Buenos Aires (parque Indoamericano) y Quito (Plaza Quitumbe).

- En la Ciudad de México no se realiza (ni siquiera como alibi) ninguna acción de espacio público en las periferias distantes ni en los barrios populares céntricos.

Ello demuestra una vez más que de nada sirve autonombrarse como gobierno de izquierda y apostar por una ciudad igualitaria, cuando en los hechos se refuerza la histórica segregación socioespacial, las grandes inversiones públicas se concentran en las partes privilegiadas de las ciudad y se sigue condenando a la periferia a la ausencia de la inversión pública.

\section{Victor Delgadillo}

Universidad Autónoma de la Ciudad de México, Colegio de Humanidades y Ciencias Sociales, Academia de Ciencia Política y Administración Urbana. Ciudad de México, México.

victor_delgadill@hotmail.com 


\section{Notas}

(1) Se trata de obras que mejoran el espacio público y la imagen urbana con el propósito de atraer inversión local e internacional.

(2) En gran medida esta experiencia fue difundida por el exilio uruguayo, es decir por los cooperativistas perseguidos por la dictadura militar.

(3) Las cooperativas Guerrero y Palo Alto en el centro y la periferia urbana respectivamente.

(4) Moscato (2000) da cuenta del rechazo de los gremios de profesionales argentinos a la inicial propuesta catalana, por lo que se organizó un concurso público local para definir el plan urbano definitivo.

(5) Esta actitud no es exclusivamente "un problema latinoamericano". Investigadores de Europa también recurren a la "importación" de conceptos en boga. Recientemente en Francia se puso de moda el concepto del gueto estadounidense para intentar "explicar" la problemática de sus barrios étnicos problemáticos ubicados en las periferias urbanas (Wacquant, 2010).

(6) No resulta ocioso mencionar un ejemplo: en julio de 2013 estudiantes españoles que volvieron a Madrid después de una estancia de intercambio en Buenos Aires, sostenían que la capital porteña con la realización de Puerto Madero se estaba "deslatinoamericanizando".

(7) Estas actividades pueden ser (co)financiados por las embajadas de países amigos.

(8) No se necesita destinar el $17 \%$ del presupuesto público territorial como ocurre en Porto Alegre, puede ser el 3\% - como se hace en la Ciudad de México - o hasta menos, cuando el objetivo es hacer creer a la población que realmente "participa" en la definición de lo público y de la política pública territorializada.

(9) Merino (2010) presenta un estado desastroso del espacio público en México: el espacio público electoral ha sido debilitado por los partidos políticos y los poderes facticos; el espacio público mediático es dominado por dos televisoras privadas; la economía mexicana (lejos de ser el lugar del intercambio y la producción) está marcada por la voracidad de los oligopolios; la clase política actúa en su propio beneficio. Por ello, Merino sostiene que el espacio público en México es un territorio secuestrado y excluyente. Aquí, los contenidos del espacio público no son negociados, sino que se han vuelto un negocio.

(10) Delgado demuestra que el espacio público es un concepto reciente que en las décadas de 1960 a 1980 casi no era utilizado. Ni Jane Jacobs, ni Jordi Borja, ni Henry Lefebvre usaban este concepto, y cuando lo mencionaban lo hacía como sinónimo de plaza y calle. 


\section{Referências}

AEP - AUTORIDAD DEL ESPACIO PÚBLICO (2012). Ciudad de México, Espacio Público 2009-2012. México DF, AEP-GDF.

ALMANDOZ, A. (coord.) (2002). Planning Latin America's Capital Cities, 1850-1950. Londres, Routledge.

ATKINSON, R. e BRIDGE, G. (coords.) (2005). Gentrification in a Global Context: the new urban colonialism. Oxon, Routledge.

BÄHR, J. e MERTINS, G. (1995). Die Lateinamerikanische Gross-Stadt, Verstädterungsprozesse und Stadtstrukturen. Darmstadt, Wissehnschaftliche Buchgesellshaft.

BORJA, J. (2003). La ciudad conquistada. Madri, Alianza Editorial.

BORJA, J. e CASTELLS, M. (1997). Local y global, la gestión de las ciudades en la era de la información. Madri, UNCHS/Taurus.

DELGADILLO, V. (2011). Patrimonio histórico y tugurios: Ias políticas habitacionales y de recuperación de los centros históricos de Buenos Aires, Ciudad de México y Quito. México, UACM.

(2013). América Latina Urbana: la construcción de un pensamiento teórico propio. Entrevista con Emilio Pradilla Cobos. Andamios, Revista de Investigación Social, v. 10, n. 22, pp. 185-201.

DELGADO, M. (2007). La Ciudad Mentirosa, fraude y miseria del "modelo Barcelona". Madri, Los Libros de la Catarata.

(2011). El espacio público como ideología. Madri, Los Libros de la Catarata.

GCABA - Gobierno de la Ciudad Autónoma de Buenos Aires (2012). Boletín del 22/6/2012. Disponível em: http://www.365buenosaires.com/buenos-aires-en-bicicleta_nota820.html

(2013). Espacio público en Buenos Aires.Disponível em: http://www.buenosaires.gob.ar/areas/ med_ambiente/. Acesso em: 31 out 2013.

GILBERT, A. (1997). La ciudad latinoamericana. México, Siglo XXI.

GOLDA, K. (2007). Retracing a relation: Barcelona's Role as Urban Model for Ibero-American Metropolises - Limas a Case Study. Trialog 93. Darmstadt, pp. 4-11.

GORMSEN, E. (1981). Die Städte in Spanischen Amerika; ein zeit-räumliches Entwicklungsmodell der letzten hundert Jahren. Erdkunde, Band 35. Bonn, pp. 290-303.

(1989). La rehabilitación de centros históricos en ciudades de América Latina y de la península ibérica. Revista Interamericana de Planificación. Guatemala, v. XXII, n. 87 e 88, pp. 233-252.

HARDOY, J. (1995). Teorías y prácticas urbanísticas en Europa entre 1850 y 1930. Su traslado a América Latina. Dana, n. 37/38, pp. 12-30.

HOFFER, A. (2003). Karl Brunner y el urbanismo europeo en América Latina. Bogotá, El Áncora Editores/ Corporación La Candelaria.

JAJAMOVICH, J. (2013). Miradas sobre intercambios internacionales y circulación intenacional de ideas y modelos urbanos. Andamios, Revista de Investigación Social, v. 10, n. 22, pp. 91-112.

MERINO, M. (coord.) (2010). ¿Qué tan público es el espacio público en México? México, FCE/Conaculta/UV. 
MOSCATO, J. (2000). “El proceso de reconversión de Puerto Madero en Buenos Aires”. In: GUTIÉRREZ, R. (coord.). La otra arquitectura: ciudad, vivienda y patrimonio. México, Conaculta.

PUIG, T. (2009). Marca Ciudad, cómo rediseñarla para asegurar un futuro espléndido para todos. Buenos Aires, Paidos.

QUEIROZ, L. R. e PECHMAN, R. (orgs.). (1996). Cidade, povo e nação. Gênese do urbanismo moderno. Rio de Janeiro, Civilização Brasileira.

RABOTNIKOF, N. (2010). “Discutiendo lo público en México”. In: MERINO, M. (coord.) ¿Qué tan público es el espacio público en México? México, FCE/Conaculta/UV.

RíOS, C. (ed.) (2008). Carlos Contreras. Revista Planificación 1927-1938. Tres Tomos, 27 números (1a edición en versión digital). México, Facultad de Arquitectura UNAM.

ROBINSON, J. (2009). Ordinary Cities. Londres, Routledge.

ROJAS, E. (ed.) (2009). Construir ciudades, mejoramiento de barrios y calidad de vida urbana. Washington DC, BID.

ROJAS, E. e De MOURA, C. (1999). Préstamos para la conservación del patrimonio histórico urbano, desafíos y oportunidades. Washington DC, BID - Departamento de Desarrollo Sostenible.

ROJAS, E.; RODRIGUEZ, E. e WEGELIN, E. (2004). Volver al Centro, la recuperación de las áreas urbanas centrales. Washington DC, BID.

SALAZAR, D. (2011). Quito un nuevo Modelo de ciudad. Folleto promocional. Trama Ediciones.

WACQUANT, L. (2010). Las dos caras de un gueto. Ensayos sobre marginalización y penalización. Buenos Aires, Siglo XXI.

Texto recebido em 20/ago/2013

Texto aprovado em 30/set/2013 
